\title{
Domain Wall Dynamics in Amorphous Microwires
}

\author{
K. Richter, Y. Kostyk, R. Varga \\ Inst. Phys., Fac. Sci., UPJS, Park Angelinum 9, 04154 Kosice, Slovakia
}

A. ZHUKOV

Dpto. Fis. Mater., Fac. Quimica, UPV/EHU, 1072, 20080, San Sebastian, Spain

\author{
AND V. LARIN
}

MFTI, Kishinev, Moldova

\begin{abstract}
Here we present the domain wall dynamics in FeNi-based microwires with positive magnetostriction. Two different ranges were found which differs by the measured domain wall mobility. At low fields, the domain wall dynamics exhibit small mobility, whereas at higher field the domain wall mobility increases. The difference in the two regimes of the domain wall dynamics is treated in terms of the different domain wall structure. At low fields, the transversal domain wall is expected, with low domain wall mobility. At high field, the vortex-type domain wall with high domain wall mobility is created.
\end{abstract}

PACS numbers: 75.60.Ej, 75.60.Jk

\section{Introduction}

The domain wall propagation is frequently used in magnetic devices (HDD, MRAM, different sensors, etc.). The speed of such devices depends on the domain wall velocity. Therefore, the study of the domain wall dynamics attracts rising interest in the last few years $[1,2]$.

Amorphous glass-coated magnetic microwires have been shown to be ideal materials for study the single domain wall dynamics due to their domain structure and corresponding magnetization process $[3,4]$. As a result of their preparation, the domain structure of microwires with positive magnetostriction consists of large single domain, which is surrounded by the radial domains.

Small closure domains appear at the ends of wire, in order to decrease the stray fields. Finally, the magnetization process in axial direction runs through 
the depining and subsequent propagation of the single domain wall in one large Barkhausen jump. This makes the microwires ideal materials for study of the single domain wall dynamics. The domain wall dynamics is described by simple linear equation [3]:

$$
v=S\left(H-H_{0}\right),
$$

where $v$ is domain wall velocity, $S$ is domain wall mobility, $H$ is an applied field and $H_{0}$ is the critical field, below which the domain wall propagation is not possible.

\section{Experimental}

We have studied the domain wall dynamics on the amorphous glass-coated microwire prepared by the Taylor-Ulitovski method. The composition of the wire was $\mathrm{Fe}_{49.6} \mathrm{Ni}_{27.9} \mathrm{Si}_{7.5} \mathrm{~B}_{15}$, the total diameter of $34.4 \mu \mathrm{m}$ and the diameter of metallic nucleus of $14.4 \mu \mathrm{m}$. The domain wall dynamics was measured by the classical Sixtus-Tonks experiments. The system consists of three coaxial coils. The primary coil of $10 \mathrm{~cm}$ length generating the exciting field was fed by $30 \mathrm{~Hz}$ frequency AC square current creating a homogeneous field along the wire of $10.5 \mathrm{~cm}$ that can be taken as static during wall propagation. Two secondary coils, symmetrically disposed at the center of the primary coils and separated by $L=6 \mathrm{~cm}$, are connected in series opposition, so two sharp opposite peaks are picked up at an oscilloscope upon passing of the propagating wall. The coils system allows us to identify the propagating wall direction whose velocity is calculated as $v=L / t$, where $t$ is the time between two maxima in the emf recorded peaks. More details can be found elsewhere $[3,4]$.

\section{Results and discussion}

The dependence of the domain wall velocity $v$ on the applied magnetic field $H$ measured in the amorphous glass-coated FeNiSiB microwire is shown in Fig. 1. Two linear regions are clearly recognized. First one, at low fields, is characterized by the lower domain wall mobility $\left(S_{t}=1.49 \mathrm{~m}^{2} /(\mathrm{A} \mathrm{s})\right)$ and negative critical propagation field $\left(H_{0}=-178,92 \mathrm{~A} / \mathrm{m}\right)$. The second one, at higher fields, is characterized by the high domain wall mobility $\left(S_{v}=16.49 \mathrm{~m}^{2} / \mathrm{A} \mathrm{s}\right)$ and positive critical propagation field $\left(H_{0}=534.24 \mathrm{~A} / \mathrm{m}\right)$. The domain wall reaches the velocities up to $8000 \mathrm{~m} / \mathrm{s}$.

The two different domain wall dynamics could be treated in terms of two different domain wall structures. As it was found theoretically [5], two different domain wall structures are possible in thin magnetic wires [6].

Firstly, it is the transversal domain wall (see Fig. 2a). Due to the specific stress distribution in the glass-coated microwires with positive magnetostriction [7], this structure is preferable at low fields. However, it creates free poles at the border between the internal and external domain structures. These free poles interact with the radial domain structure, which results in the smaller domain wall mobility. The negative critical propagation field can be explained by the fact that 


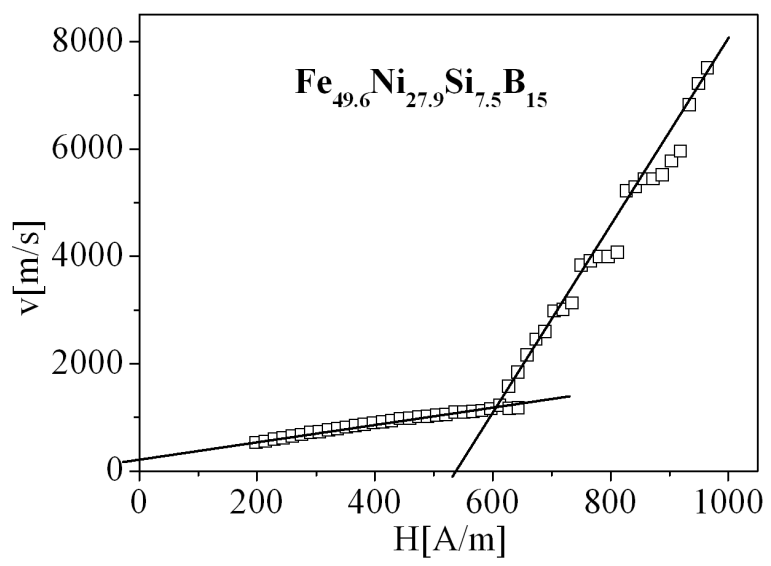

Fig. 1. The domain wall velocity $v$ dependence on the applied magnetic field $H$.

the closure domain wall with the transversal domain structure already exists in microwires even without applied magnetic field [4].

a)

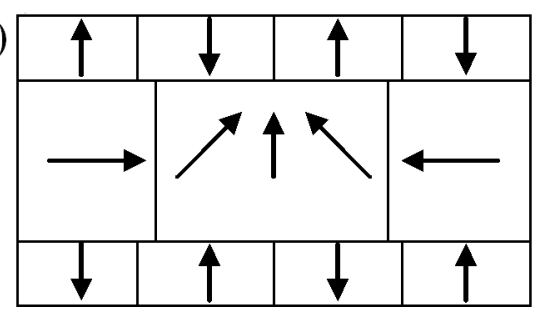

b)

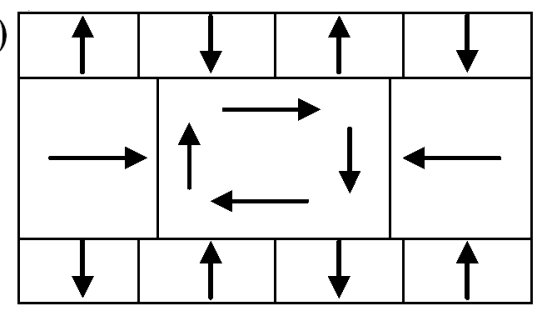

Fig. 2. Schematic structure of transversal- (a) and vortex-type domain wall (b) in magnetic microwire.

Applying the high amplitude of the square shaped magnetic field during our experiment, the rise-time of the field is very short. The transversal domain wall has no time to depin. Instead, the vortex-type domain wall nucleates at the end of the microwire. Its critical propagation field is positive and can be estimated from the linear fit according to Eq. (1). The energy of the vortex-type domain wall is much higher than transversal ones, since the exchange is higher. Therefore, it does not appear at low field. However, the vortex-type domain wall does not create the free poles at its surface and it can propagate without interaction with the radial domain structure below the surface. Therefore, its domain wall mobility is higher and it can reach very high velocities - up to $8000 \mathrm{~m} / \mathrm{s}$ in our case. Such a domain wall is very fast, faster than the sound velocity in magnetic microwires $(\approx 4700 \mathrm{~m} / \mathrm{s}[8])$. Therefore, the observed domain wall is supersonic.

As it was shown theoretically, once the domain wall propagates, it does not change the type [5]. In the intermediate region $(H \approx 600-650 \mathrm{~m} / \mathrm{s})$, both domain wall types can appear. 


\section{Conclusions}

We have studied the single domain wall dynamics in amorphous glass-coated microwires. Two different domain wall dynamics regimes have been found. First one is connected to the transversal domain wall with lower mobility and negative critical field. The second one is characterized by the higher domain wall mobility and high velocities up to $8000 \mathrm{~m} / \mathrm{s}$ and is ascribed to the vortex type domain wall.

\section{Acknowledgments}

This work was supported by the scientific grants APVT-20-007804 and VEGA No. 1/3035/06.

\section{References}

[1] G.S.D. Beach, C. Nistor, C. Knutson, M. Tsoi, J.L. Erskine, Nature Mater. 4, 741 (2005).

[2] D. Atkinson, D.A. Allwood, G. Xiong, M.D. Cooke, C.C. Faulkner, R.P. Cowburn, Nature Mater. 2, 85 (2003).

[3] R. Varga, K.L. Garcia, M. Vázquez, P. Vojtanik, Phys. Rev. Lett. 94, 017201 (2005).

[4] R. Varga, A. Zhukov, J.M. Blanco, M. Ipatov, V. Zhukova, J. Gonzalez, P. Vojtaník, Phys. Rev. B 74, 212405 (2006).

[5] R.D. McMichael, M.J. Donahue, IEEE Trans. Magn. 33, 4167 (1997).

[6] R. Cowburn, D.E. Petit, Nature Mater. 4, 884 (2005).

[7] H. Chiriac, T.A. Ovari, G. Pop, Phys. Rev. B 52, 10104 (1995).

[8] H. Chiriac, E. Hristoforou, M. Neagu, I. Darie, A.E. Moga, J. Non-Cryst. Solids 287, 413 (2001). 